


\title{
Water Quality Parameters for the Culture of Rainbow Trout, Oncorhynchus mykiss (Walbaum) in the Raceways of Kathmandu, Nepal
}

\author{
Rakesh Prasad Bhagat ${ }^{1,2} *$ and Sudip Barat ${ }^{2}$ \\ ${ }^{I}$ Department of Zoology, Trichandra Multiple Campus, Tribhuvan University, Kathmandu, Nepal \\ ${ }^{2}$ Aquaculture and Limnology Research Unit, Department of Zoology, University of North Bengal, Siliguri- \\ 734013, District Darjeeling, West Bengal, India \\ *e-mail: rakesh.bhagat2009@gmail.com
}

Received: 23.08.2015; Accepted: 14.11.2015

\begin{abstract}
Eighteen water quality parameters ( 5 physical, 9 chemical, 2 climatic and 2 geographical) were investigated for the water quality assessment so as to know whether water in the raceways from spring-fed torrential stream at a high altitude was feasible and suitable for the culture of rainbow trout, Oncorhynchus mykiss (Walbaum) or not. Results indicated colourless, odourless, and crystalclear water throughout the year with air temperature ranging from 11.4-26.9 $\left(20.3 \pm 1.1^{\circ} \mathrm{C}\right)$, water temperature 8.4-21.5 $\left(16 \pm 1.1^{\circ} \mathrm{C}\right)$, water velocity 1.5-3.5 $\left(2.5 \pm 0.11 \mathrm{~m} \mathrm{sec}^{-1}\right)$, water discharge 37-92 $\left(56 \pm 2.87 \mathrm{~L} \mathrm{sec}^{-1}\right)$, turbidity 3-19 $(11 \pm 1.04 \mathrm{NTU}), \mathrm{pH}$ 6.5-8.2 $(7.44 \pm 0.11)$, electrical conductivity $35-$ $204\left(112.13 \pm 11.2 \mu \mathrm{S} \mathrm{cm}^{-1}\right)$, dissolved oxygen 5.9-10.5 $\left(8.2 \pm 0.3 \mathrm{mg} \mathrm{L}^{-1}\right)$, free carbon dioxide 1.4-5.1 $\left(3.6 \pm 0.2 \mathrm{mg} \mathrm{L}^{-1}\right)$, total alkalinity 17-97 $\left(55.1 \pm 5.32 \mathrm{mg} \mathrm{L}^{-1}\right)$, total hardness $11-90\left(47 \pm 5.06 \mathrm{mg} \mathrm{L}^{-1}\right)$, phosphate-P 0.01-0.50 $\left(0.14 \pm 0.02 \mathrm{mg} \mathrm{L}^{-1}\right)$, ammonium-N $0.09-0.91\left(0.28 \pm 0.04 \mathrm{mg} \mathrm{L}^{-1}\right)$, nitrate-N $0.01-0.83\left(0.17 \pm 0.04 \mathrm{mg} \mathrm{L}^{-1}\right)$, relative humidity $62.4-88.7(75.01 \pm 1.59 \%)$, rainfall $0.0-503.4$ $(132.44 \pm 32.83 \mathrm{~mm})$, altitude $1550 \mathrm{msl}$, and water resource stream-fed torrential stream. Correlation analyses of the parameters showed strongest correlation at the significance level of 0.01 . All parameters were positively correlated except $\mathrm{pH}$, electrical conductivity, dissolved oxygen, total alkalinity, and total hardness which were negatively correlated with rest. Parameters of the first year were slightly higher than second due to fluctuation in temperature, velocity and discharge, relative humidity, and rainfall influenced by climatic factors, geography, seasons, and environment of the origin and occurrence of the water resource, thus affecting rest of the parameters. Water velocity and water discharge could be maintained as per requirement of the culture. These parameters were within permissible limits being feasible and suitable for rainbow trout culture.
\end{abstract}

Key words: Rainbow trout culture, raceways, water quality assessment

\section{Introduction}

Rainbow trout, Oncorhynchus mykiss (Walbaum) requires cold and crystal-clear running water with low water temperature, high dissolved oxygen, moderate free carbon dioxide, proper water velocity and balanced water discharge (Huet, 1975). In addition, it requires optimum $\mathrm{pH}$, electrical conductivity, turbidity, total alkalinity, total hardness, $\mathrm{NH}_{4}, \mathrm{NO}_{3}$ and $\mathrm{PO}_{4}$ (Bardach et al.,
1972). Furthermore, its culture is to be supported by some climatic factors like relative humidity and rainfall and geographical factors like altitude andwater resource. Such condition of ever running water could be met with running water habitat of raceways if supplied with perennial and dependable water resource like spring-fed torrential stream. 
Pradhan et al. (2007/2008) studied on water quality parameters in the raceways of Godawari, Kathmandu, Nepal. The present study aimed to assess feasibility and suitability of the water quality parameters of the raceways at Kakani, Kathmandu, Nepal for the culture of rainbow trout.

\section{Materials and methods \\ Study site}

The raceways supplied with perennial spring-fed torrential stream lie in Kakani, Nuwakot district, Kathmandu valley, Nepal at a high altitude of $1550 \mathrm{msl}$ situated at latitude of $27^{\circ} 48^{\prime} \mathrm{N}$ and longitude $85^{\circ} 15^{\prime} \mathrm{E}$.

The study was conducted from June 2010 to May 2012 at monthly intervals representing four seasons: monsoon (June to August), autumn (September to November), winter (December to February) and summer (March to May).Water velocity was determined by recording time to travel $10 \mathrm{~m}$ distance using a float (an orange coloured cork) in the feeding channel of raceways (Adoni et al., 1985). Water discharge was determined by measuring time to fill a 1001 plastic tank. Turbidity was determined by turbidometer (model 2001A). pH and electrical conductivity were measured by pocket $\mathrm{pH}$ meter and conductivity meter (211-Microprocessor), respectively.

Water samples were collected in between 8:00 a.m. to 9:00 a.m. in the morning. Dissolved oxygen, free carbon dioxide, total alkalinity, total hardness, $\mathrm{NH}_{4}, \mathrm{NO}_{3}$, and $\mathrm{PO}_{4}$ were determined by as per APHA (2005) and preserved as per Svobodova et al. (1993) for further analyses in the laboratory within 24 hours.

\section{Results and discussions}

Rainbow trout require water temperature $10-18^{\circ} \mathrm{C}$ (Yamazaki, 1991), pH 6.5-8.5, and dissolved oxygen above $8 \mathrm{mg}^{-1}$ (Huet, 1975). Water quality parameters are represented as monsoon (June to August), autumn (September to November), winter (December to February) and summer (March to May) in Table 1. All the parameters were within permissible limits for the culture of rainbow trout.

The range of air temperature and water temperature were $11.4-26.9^{\circ} \mathrm{C}$ and 8.4 $21.5^{\circ} \mathrm{C}$ respectively. Monsoon was hot and winter was cold in both the years. June was the hottest and January was the coldest month. McGregor and Nieuwolt (1998) reported $0.65^{\circ} \mathrm{C}$ decrease in air temperature per $100 \mathrm{~m}$ increase in altitude. Air temperature is related with water temperature and it higher than water temperature (APHA, 2005; Arain et al., 2009).

Water temperature was depended on air temperature (Manon and Hossain, 2011) and altitude (Jacobsen, 2008). Acherjee and Barat (2011) reported $0.6^{\circ} \mathrm{C}$ decrease in water temperature per $100 \mathrm{~m}$ increase in altitude.

Water velocity and water discharge ranged $1.5-3.5 \mathrm{~m} \mathrm{sec}^{-1}$ and $37-921 \mathrm{sec}^{-1}$ respectively, having maximum in monsoon and minimum in winter season. Similar seasonal trend was recorded for turbidity which ranged from 3-19. High water velocity and water discharge during monsoon and low during winter was due to rainfall (Wetzel, 2001). Acherjee and Barat (2011) reported $0.61-1.5 \mathrm{~m} \mathrm{sec}^{-1}$ with its lowest value in November and highest in July. Bartoli et al., (2007) reported 1901 $\mathrm{sec}^{-1}$ water discharge in rainbow trout raceways and suitable value for 10,000 incubated eggs of rainbow trout was 1201 $\sec ^{-1}$. 
Rakesh Prasad Bhagat and Sudip Barat / Our Nature (2015), 13(1): 50-57.

Table 1. Season-wise, annual and two-year data of water quality parameters of the raceways at Kakani, Kathmandu, Nepal with min, max and mean \pm SE.

\begin{tabular}{|c|c|c|c|c|c|}
\hline \multirow[t]{2}{*}{ Parameters } & \multicolumn{5}{|c|}{ June 2010 to May 2011} \\
\hline & \begin{tabular}{lr}
\multicolumn{2}{|l|}{ Monsoon } \\
(June & 2010 \\
to & August \\
$2010)$ &
\end{tabular} & $\begin{array}{l}\text { Autumn } \\
\text { (September } \\
2010 \text { to } \\
\text { November } \\
2010)\end{array}$ & $\begin{array}{l}\text { Winter } \\
\text { (December } \\
2010 \quad \text { to } \\
\text { February } \\
\text { 2011) }\end{array}$ & $\begin{array}{l}\text { Summer } \\
\text { (March 2011 } \\
\text { to May 2011) }\end{array}$ & $\begin{array}{l}\text { Annual } \\
\text { (June 2010 } \\
\text { to May 2011) }\end{array}$ \\
\hline $\begin{array}{l}\text { Air } \\
\text { temperature }\end{array}$ & $\begin{array}{l}26.3-26.9 \\
26.57 \pm 0.18\end{array}$ & $\begin{array}{l}16.8-25.7 \\
21.43 \pm 2.58\end{array}$ & $\begin{array}{l}11.7-13.9 \\
12.67 \pm 0.65\end{array}$ & $\begin{array}{l}18.2-23.2 \\
20.9 \pm 1.5\end{array}$ & $\begin{array}{l}11.7-26.9 \\
20.4 \pm 1.6 \\
\end{array}$ \\
\hline $\begin{array}{l}\text { Water } \\
\text { temperature }\end{array}$ & $\begin{array}{l}21.2-21.5 \\
21.33 \pm 0.09\end{array}$ & $\begin{array}{l}13.1-20.7 \\
16.9 \pm 2.19\end{array}$ & $\begin{array}{l}8.6-10.5 \\
9.4 \pm 0.57\end{array}$ & $\begin{array}{l}13.8-18.8 \\
16.17 \pm 1.28\end{array}$ & $\begin{array}{l}8.6-21.5 \\
16.0 \pm 1.4\end{array}$ \\
\hline Water velocity & $\begin{array}{l}2.7-3.0 \\
2.87 \pm 0.09\end{array}$ & $\begin{array}{l}1.8-2.8 \\
2.23 \pm 0.30\end{array}$ & $\begin{array}{l}1.5-2.2 \\
1.93 \pm 0.22\end{array}$ & $\begin{array}{l}1.9-2.5 \\
2.23 \pm 0.18\end{array}$ & $\begin{array}{l}1.5-3.0 \\
2.32 \pm 0.14\end{array}$ \\
\hline $\begin{array}{l}\text { Water } \\
\text { discharge }\end{array}$ & $\begin{array}{l}60-84 \\
70.33 \pm 7.13\end{array}$ & $\begin{array}{l}41-66 \\
51.67 \pm 7.45\end{array}$ & $\begin{array}{l}37-50 \\
43.67 \pm 3.76\end{array}$ & $\begin{array}{l}43-59 \\
51 \pm 4.62 \\
\end{array}$ & $\begin{array}{l}37-84 \\
54.17 \pm 3.91\end{array}$ \\
\hline Turbidity & $\begin{array}{l}14-18 \\
16.33 \pm 1.20\end{array}$ & $\begin{array}{l}4-15 \\
10.33 \pm 3.28\end{array}$ & $\begin{array}{l}3-6 \\
4.67 \pm 0.88\end{array}$ & $\begin{array}{l}7-11 \\
9 \pm 1.16\end{array}$ & $\begin{array}{l}3-18 \\
10.08 \pm 1.50\end{array}$ \\
\hline $\mathrm{pH}$ & $\begin{array}{l}6.5-6.8 \\
6.63 \pm 0.09\end{array}$ & $\begin{array}{l}7.1-8.0 \\
7.57 \pm 0.26\end{array}$ & $\begin{array}{l}7.9-8.1 \\
8.0 \pm 0.06\end{array}$ & $\begin{array}{l}7.0-7.8 \\
7.33 \pm 0.24\end{array}$ & $\begin{array}{l}6.5-8.1 \\
7.38 \pm 0.17\end{array}$ \\
\hline $\begin{array}{l}\text { Electrical } \\
\text { conductivity }\end{array}$ & $\begin{array}{l}35-72 \\
52.67 \pm 10.71\end{array}$ & $\begin{array}{l}103-166 \\
135.33 \pm 18.21\end{array}$ & $\begin{array}{l}149-200 \\
173.67 \pm 14.75\end{array}$ & $\begin{array}{l}50-98 \\
69.67 \pm 14.52\end{array}$ & $\begin{array}{l}35-200 \\
107.83 \pm 16.05\end{array}$ \\
\hline $\begin{array}{l}\text { Dissolved } \\
\text { oxygen }\end{array}$ & $\begin{array}{l}5.9-7.2 \\
6.63 \pm 0.38\end{array}$ & $\begin{array}{l}8.5-9.8 \\
9.2 \pm 0.38\end{array}$ & $\begin{array}{l}9.5-10.3 \\
9.97 \pm 0.24\end{array}$ & $\begin{array}{l}6.5-8.4 \\
7.37 \pm 0.54\end{array}$ & $\begin{array}{l}5.9-10.3 \\
8.29 \pm 0.44\end{array}$ \\
\hline $\begin{array}{l}\text { Free carbon } \\
\text { dioxide }\end{array}$ & $\begin{array}{l}4.4-4.9 \\
4.63 \pm 0.14 \\
\end{array}$ & $\begin{array}{l}2.9-3.9 \\
3.43 \pm 0.29 \\
\end{array}$ & $\begin{array}{l}1.4-2.3 \\
1.83 \pm 0.26 \\
\end{array}$ & $\begin{array}{l}3.1-4.2 \\
3.73 \pm 0.33\end{array}$ & $\begin{array}{l}1.4-4.9 \\
3.41 \pm 0.33\end{array}$ \\
\hline Total alkalinity & $\begin{array}{l}17-38 \\
27 \pm 6.08 \\
\end{array}$ & $\begin{array}{l}53-80 \\
66.67 \pm 7.80\end{array}$ & $\begin{array}{l}69-96 \\
82.33 \pm 7.80\end{array}$ & $\begin{array}{l}21-65 \\
46.33 \pm 13.13\end{array}$ & $\begin{array}{l}17-96 \\
55.58 \pm 7.39\end{array}$ \\
\hline Total hardness & $\begin{array}{l}11-31 \\
20.67 \pm 5.78\end{array}$ & $\begin{array}{l}42-70 \\
56.67 \pm 8.11\end{array}$ & $\begin{array}{l}57-88 \\
72 \pm 8.96\end{array}$ & $\begin{array}{l}15-60 \\
39 \pm 13.08\end{array}$ & $\begin{array}{l}11-88 \\
47.08 \pm 7.03\end{array}$ \\
\hline Phosphate-P & $\begin{array}{l}0.18-0.26 \\
0.22 \pm 0.02\end{array}$ & $\begin{array}{l}0.07-0.20 \\
0.14 \pm 0.04\end{array}$ & $\begin{array}{l}0.01-0.08 \\
0.04 \pm 0.02\end{array}$ & $\begin{array}{l}0.09-0.18 \\
0.13 \pm 0.03\end{array}$ & $\begin{array}{l}0.01-0.26 \\
0.13 \pm 0.02\end{array}$ \\
\hline Ammonium-N & $\begin{array}{l}0.29-0.91 \\
0.51 \pm 0.20\end{array}$ & $\begin{array}{l}0.12-0.21 \\
0.18 \pm 0.03\end{array}$ & $\begin{array}{l}0.09-0.16 \\
0.12 \pm 0.02\end{array}$ & $\begin{array}{l}0.14-0.37 \\
0.27 \pm 0.07\end{array}$ & $\begin{array}{l}0.09-0.91 \\
0.27 \pm 0.06\end{array}$ \\
\hline Nitrate-N & $\begin{array}{l}0.23-0.83 \\
0.49 \pm 0.18 \\
\end{array}$ & $\begin{array}{l}0.02-0.38 \\
0.15 \pm 0.12 \\
\end{array}$ & $\begin{array}{l}0.01-0.06 \\
0.03 \pm 0.01 \\
\end{array}$ & $\begin{array}{l}0.04-0.19 \\
0.11 \pm 0.04\end{array}$ & $\begin{array}{l}0.01-0.83 \\
0.20 \pm 0.07 \\
\end{array}$ \\
\hline $\begin{array}{l}\text { Relative } \\
\text { humidity }\end{array}$ & $\begin{array}{l}71.1-88.7 \\
81.23 \pm 95.25\end{array}$ & $\begin{array}{l}79.7-84.6 \\
81.67 \pm 1.50\end{array}$ & $\begin{array}{l}69.9-76.5 \\
73.4 \pm 1.92\end{array}$ & $\begin{array}{l}63.2-66.3 \\
65.1 \pm 0.96\end{array}$ & $\begin{array}{l}63.2-88.7 \\
75.35 \pm 2.39\end{array}$ \\
\hline Rainfall & $\begin{array}{l}145.4-402.6 \\
296.93 \pm 77.71\end{array}$ & $\begin{array}{l}0.0-272.8 \\
101.47 \pm 86.15\end{array}$ & $\begin{array}{l}0.0-50.0 \\
18.4 \pm 15.87\end{array}$ & $\begin{array}{l}8.3-68.4 \\
48.33 \pm 20.02\end{array}$ & $\begin{array}{l}0.0-402.6 \\
116.28 \pm 41.36 \\
\end{array}$ \\
\hline
\end{tabular}




\begin{tabular}{|c|c|c|c|c|c|}
\hline \multicolumn{5}{|c|}{ June 2011 to May 2012} & \multirow{2}{*}{$\begin{array}{l}\text { Remarks } \\
\text { Two years } \\
\text { (June 2010 to } \\
\text { May 2012) }\end{array}$} \\
\hline $\begin{array}{l}\text { Monsoon } \\
\text { (June 2011 to } \\
\text { August 2011) }\end{array}$ & $\begin{array}{l}\text { Autumn } \\
\text { (September } \\
2011 \text { to } \\
\text { November } \\
\text { 2011) }\end{array}$ & $\begin{array}{l}\text { Winter } \\
\text { (December } \\
2011 \text { to } \\
\text { February } \\
\text { 2012) }\end{array}$ & $\begin{array}{l}\text { Summer } \\
\text { (March 2012 } \\
\text { to May 2012) }\end{array}$ & $\begin{array}{l}\text { Annual } \\
\text { (June 2011 } \\
\text { to May 2012) }\end{array}$ & \\
\hline $25.7-26.1$ & $19.4-24.8$ & $11.4-13.2$ & $17.1-23.4$ & $11.4-26.1$ & $11.4-26.9$ \\
\hline $25.9 \pm 0.12$ & $21.83 \pm 1.58$ & $12.57 \pm 0.84$ & $20.47 \pm 1.83$ & $20.18 \pm 1.55$ & $20.3 \pm 1.1$ \\
\hline $21.1-21.3$ & $12.2-20.1$ & $8.4-9.9$ & $13.4-18.2$ & $8.4-21.3$ & $8.4-21.5$ \\
\hline $21.2 \pm 0.06$ & $17.33 \pm 2.57$ & $9.03 \pm 0.45$ & $16.13 \pm 1.42$ & $16.05 \pm 1.47$ & $16 \pm 1.1$ \\
\hline $3.2-3.5$ & $1.8-3.0$ & $1.6-2.5$ & $2.0-2.8$ & $1.6-3.5$ & $1.5-3.5$ \\
\hline $3.33 \pm 0.09$ & $2.37 \pm 0.35$ & $2.17 \pm 0.29$ & $2.43 \pm 0.23$ & $2.61 \pm 0.18$ & $2.5 \pm 0.11$ \\
\hline $69-92$ & $42-62$ & $39-53$ & $45-59$ & $39-92$ & $37-92$ \\
\hline $77.67 \pm 7.22$ & $50.67 \pm 5.93$ & $47.67 \pm 4.37$ & $53 \pm 4.16$ & $57.42 \pm 4.32$ & $56 \pm 2.87$ \\
\hline $15-19$ & $5-16$ & $4-7$ & $9-11$ & $4-19$ & $3-19$ \\
\hline $17.33 \pm 1.20$ & $11.33 \pm 3.28$ & $5.67 \pm 0.88$ & $10 \pm 0.6$ & $11.17 \pm 1.48$ & $11 \pm 1.04$ \\
\hline $6.7-7.2$ & $7.4-7.9$ & $8.0-8.2$ & $7.2-7.6$ & $6.7-8.2$ & $6.5-8.2$ \\
\hline $6.9 \pm 0.15$ & $7.6 \pm 0.15$ & $8.1 \pm 0.06$ & $7.4 \pm 0.1$ & $7.5 \pm 0.14$ & $7.44 \pm 0.11$ \\
\hline $40-80$ & $121-172$ & $157-204$ & $52-109$ & $40-204$ & $35-204$ \\
\hline $62 \pm 11.72$ & $147.67 \pm 14.77$ & $181.33 \pm 13.59$ & $74.67 \pm 17.46$ & $116.42 \pm 16.23$ & $112.13 \pm 11.2$ \\
\hline $6.1-7.1$ & $8.2-9.5$ & $9.0-10.5$ & $6.6-8.0$ & $6.1-10.5$ & $5.9-10.5$ \\
\hline $6.5 \pm 0.31$ & $8.87 \pm 0.38$ & $9.83 \pm 0.44$ & $7.2 \pm 0.42$ & $8.1 \pm 0.43$ & $8.2 \pm 0.3$ \\
\hline $3.8-5.1$ & $2.9-3.5$ & $2.5-3.7$ & $4.2-4.5$ & $2.5-5.1$ & $1.4-5.1$ \\
\hline $4.57-0.39$ & $3.2 \pm 0.17$ & $2.97 \pm 0.37$ & $4.37 \pm 0.09$ & $3.78 \pm 0.24$ & $3.6 \pm 0.2$ \\
\hline $20-41$ & $55-82$ & $75-97$ & $22-44$ & $20-97$ & $17-97$ \\
\hline $29.67 \pm 6.12$ & $69 \pm 7.81$ & $87.67 \pm 6.57$ & $32 \pm 6.43$ & $54.58 \pm 7.98$ & $55.1 \pm 5.32$ \\
\hline $13-34$ & $45-75$ & $65-90$ & $16-37$ & $13-90$ & $11-90$ \\
\hline $23 \pm 6.08$ & $60 \pm 8.66$ & $78 \pm 7.23$ & $26.33-6.07$ & $46.83 \pm 7.59$ & $47 \pm 5.06$ \\
\hline $0.19-0.50$ & $0.04-0.21$ & $0.01-0.09$ & $0.07-0.14$ & $0.01-0.50$ & $0.01-0.50$ \\
\hline $0.32 \pm 0.09$ & $0.11 \pm 0.05$ & $0.04 \pm 0.02$ & $0.11 \pm 0.02$ & $0.15 \pm 0.04$ & $0.14 \pm 0.02$ \\
\hline $0.23-0.80$ & $0.21-0.27$ & $0.16-0.22$ & $0.21-0.37$ & $0.16-0.80$ & $0.09-0.91$ \\
\hline $0.47 \pm 0.17$ & $0.24 \pm 0.02$ & $0.18 \pm 0.02$ & $0.28 \pm 0.05$ & $0.29 \pm 0.05$ & $0.28 \pm 0.04$ \\
\hline $0.21-0.45$ & $0.03-0.24$ & $0.02-0.10$ & $0.04-0.18$ & $0.02-0.45$ & $0.01-0.83$ \\
\hline $0.32 \pm 0.07$ & $0.12 \pm 0.06$ & $0.06 \pm 0.02$ & $0.11 \pm 0.04$ & $0.15 \pm 0.04$ & $0.17 \pm 0.04$ \\
\hline $77.8-85.4$ & $74.0-84.8$ & $68.2-75.1$ & $62.4-66.3$ & $62.4-85.4$ & $62.4-88.7$ \\
\hline $82.53 \pm 2.38$ & $78.17 \pm 3.35$ & $71.93 \pm 2.01$ & $64.4 \pm 1.13$ & $74.68 \pm 2.20$ & $75.01 \pm 1.59$ \\
\hline $256.0-503.4$ & $6.8-294.1$ & $0.0-42.2$ & $19.4-86.4$ & $0.0-503.4$ & $0.0-503.4$ \\
\hline $404.67 \pm 75.66$ & $108.63 \pm 92.89$ & $23.03 \pm 12.34$ & $50.07 \pm 20.02$ & $148.6 \pm 52.43$ & $132.44 \pm 32.83$ \\
\hline
\end{tabular}


Rakesh Prasad Bhagat and Sudip Barat / Our Nature (2015), 13(1): 50-57.

Table 2. Pearson correlation coefficient along with significance (two-tailed) of physico-chemical parameters of the raceways at Kakani, Kathmandu, Nepal

\begin{tabular}{|c|c|c|c|c|c|c|}
\hline Parameters & AT & WT & WV & WD & TBD & $\mathrm{pH}$ \\
\hline Air temperature & 1 & & & & & \\
\hline Water temperature & $\begin{array}{l}0.999^{* *} \\
0.000\end{array}$ & 1 & & & & \\
\hline Water velocity & $\begin{array}{l}0.777 * * \\
0.000\end{array}$ & $\begin{array}{l}0.799 * * \\
0.000\end{array}$ & 1 & & & \\
\hline Water discharge & $\begin{array}{l}0.772 * * \\
0.000\end{array}$ & $\begin{array}{l}0.787 * * \\
0.000\end{array}$ & $\begin{array}{l}0.925 * * \\
0.000\end{array}$ & 1 & & \\
\hline Turbidity & $\begin{array}{l}0.921^{* *} \\
0.000\end{array}$ & $\begin{array}{l}0.928^{* *} \\
0.000\end{array}$ & $\begin{array}{l}0.886^{* *} \\
0.000\end{array}$ & $\begin{array}{l}0.890 \text { ** } \\
0.000\end{array}$ & 1 & \\
\hline $\mathrm{pH}$ & $\begin{array}{l}-0.931^{* *} \\
0.000\end{array}$ & $\begin{array}{l}-0.933^{* *} \\
0.000\end{array}$ & $\begin{array}{l}-757 * * \\
0.000 \\
\end{array}$ & $\begin{array}{l}-0.736^{* *} \\
0.000\end{array}$ & $\begin{array}{l}-0.854 * * \\
0.000\end{array}$ & 1 \\
\hline Electrical conductivity & $\begin{array}{l}-0.850 * * \\
0.000\end{array}$ & $\begin{array}{l}-0.850^{* *} \\
0.000\end{array}$ & $\begin{array}{l}-678 * * \\
0.000\end{array}$ & $\begin{array}{l}-0.638^{* *} \\
0.001\end{array}$ & $\begin{array}{l}-0.711 * * \\
0.000\end{array}$ & $\begin{array}{l}0.910 * * \\
0.000\end{array}$ \\
\hline Dissolved oxygen & $\begin{array}{l}-0.845^{* *} \\
0.000\end{array}$ & $\begin{array}{l}-0.848^{* *} \\
0.000\end{array}$ & $\begin{array}{l}-741 * * \\
0.000\end{array}$ & $\begin{array}{l}-0.678^{* *} \\
0.000\end{array}$ & $\begin{array}{l}-0.746^{* *} \\
0.000\end{array}$ & $\begin{array}{l}0.915^{* *} \\
0.000\end{array}$ \\
\hline Free carbon dioxide & $\begin{array}{l}0.827^{* *} \\
0.000\end{array}$ & $\begin{array}{l}0.829^{* *} \\
0.000\end{array}$ & $\begin{array}{l}0.701^{* *} \\
0.000\end{array}$ & $\begin{array}{l}0.637^{* *} \\
0.001\end{array}$ & $\begin{array}{l}0.750^{* *} \\
0.000\end{array}$ & $\begin{array}{l}0.864^{* *} \\
0.000\end{array}$ \\
\hline Total alkalinity & $\begin{array}{l}-0.848^{* *} \\
0.000\end{array}$ & $\begin{array}{l}-0.852^{* *} \\
0.000\end{array}$ & $\begin{array}{l}-0.718^{*} \\
0.000\end{array}$ & $\begin{array}{l}-0.658^{* *} \\
0.000\end{array}$ & $\begin{array}{l}-0.739^{* *} \\
0.000\end{array}$ & $\begin{array}{l}0.912 * * \\
0.000\end{array}$ \\
\hline Total hardness & $\begin{array}{l}-0.853 * * \\
0.000\end{array}$ & $\begin{array}{l}-0.858^{* *} \\
0.000\end{array}$ & $\begin{array}{l}-0.715^{* *} \\
0.001\end{array}$ & $\begin{array}{l}-0.659 * * \\
0.001\end{array}$ & $\begin{array}{l}-0.744 * * \\
0.003\end{array}$ & $\begin{array}{l}0.917 * * \\
0.000\end{array}$ \\
\hline Phosphate-P & $\begin{array}{l}0.771 * * \\
0.000\end{array}$ & $\begin{array}{l}0.782 * * \\
0.000\end{array}$ & $\begin{array}{l}0.818^{* *} \\
0.000\end{array}$ & $\begin{array}{l}0.903^{* *} \\
0.000\end{array}$ & $\begin{array}{l}0.853^{* *} \\
0.000\end{array}$ & $\begin{array}{l}-0.667^{*} \\
0.010\end{array}$ \\
\hline Ammonium-N & $\begin{array}{l}0.557^{* *} \\
0.005\end{array}$ & $\begin{array}{l}0.555^{* *} \\
0.005\end{array}$ & $\begin{array}{l}0.489^{*} \\
0.015\end{array}$ & $\begin{array}{l}0.378 \\
0.069 \\
\end{array}$ & $\begin{array}{l}0.480^{*} \\
0.018\end{array}$ & $\begin{array}{l}-0.661 * * \\
0.000\end{array}$ \\
\hline Nitrate-N & $\begin{array}{l}0.704^{* *} \\
0.000\end{array}$ & $\begin{array}{l}0.708^{* *} \\
0.000\end{array}$ & $\begin{array}{l}0.721^{* *} \\
0.000\end{array}$ & $\begin{array}{l}0.884^{* *} \\
0.000\end{array}$ & $\begin{array}{l}0.797 * * \\
0.000\end{array}$ & $\begin{array}{l}-0.689^{* *} \\
0.000\end{array}$ \\
\hline Relative humidity & $\begin{array}{l}0.425 * \\
0.038 \\
\end{array}$ & $\begin{array}{l}0.433^{*} \\
0.035\end{array}$ & $\begin{array}{l}0.439^{*} \\
0.320\end{array}$ & $\begin{array}{l}0.558 * * \\
0.005\end{array}$ & $\begin{array}{l}0.563 * * \\
0.004\end{array}$ & $\begin{array}{l}-0.303 \\
0.149\end{array}$ \\
\hline Rainfall & $\begin{array}{l}0.758^{* *} \\
0.000\end{array}$ & $\begin{array}{l}0.773 * * \\
0.000\end{array}$ & $\begin{array}{l}0.862 * * \\
0.000\end{array}$ & $\begin{array}{l}0.932 * * \\
0.000\end{array}$ & $\begin{array}{l}0.886^{* *} \\
0.000\end{array}$ & $\begin{array}{l}-0.711^{* *} \\
0.004\end{array}$ \\
\hline
\end{tabular}




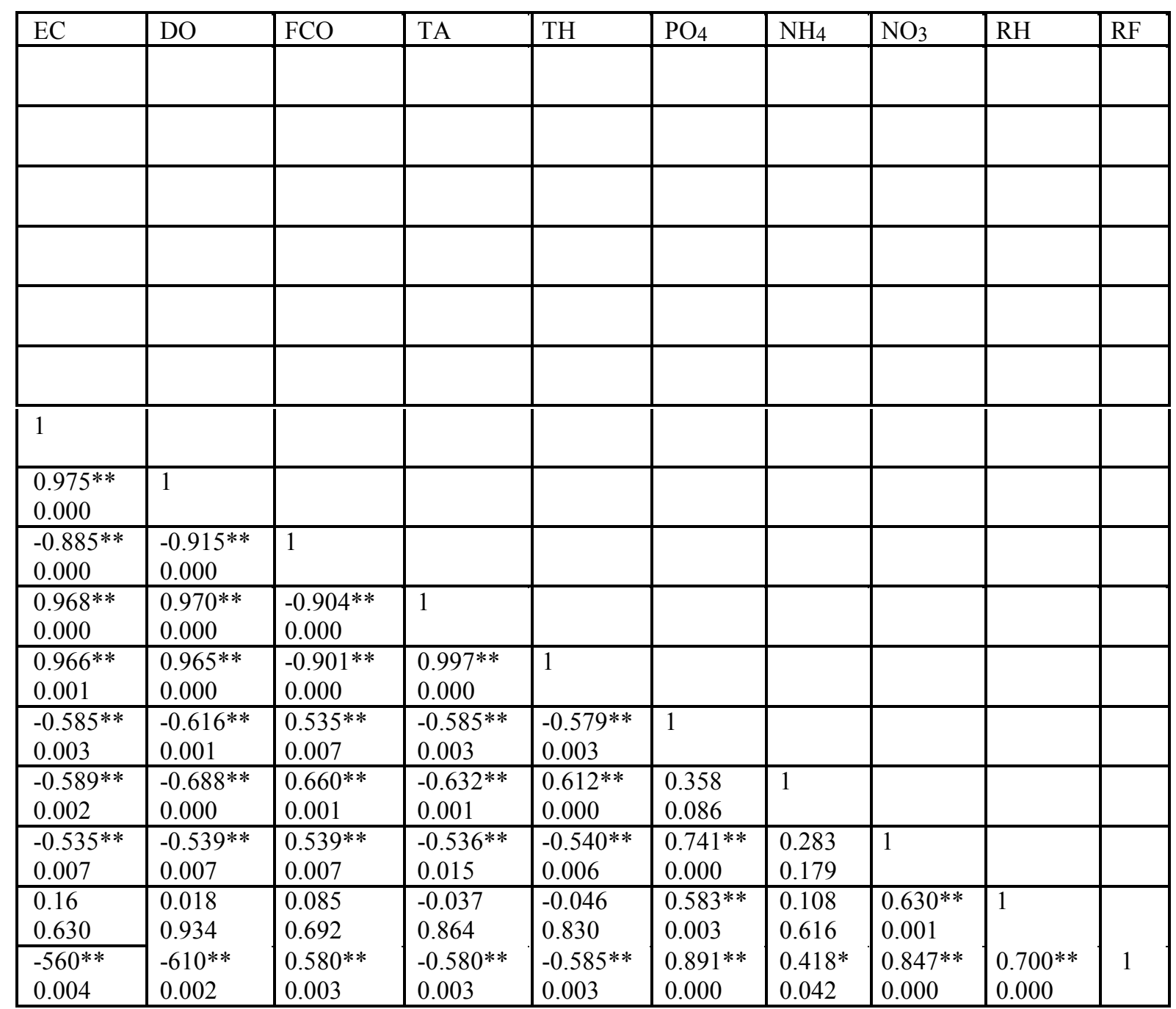


Winter had maximum $\mathrm{pH}$, electrical conductivity and dissolved oxygen than monsoon season that ranged 6.5-8.2, 35-204 $\mu \mathrm{S} \mathrm{cm}{ }^{-1}$ and 5.9-10.5 $\mathrm{mg} \mathrm{l}^{-1}$ respectively. However, free carbon dioxide, $\mathrm{PO}_{4}, \mathrm{NH}_{4}$, and $\mathrm{NO}_{3}$ was maximum during summer and minimum during winter that ranged 1.4-5.1 $\mathrm{mg} \mathrm{l}^{-1}, 0.01-0.50 \mathrm{mg} \mathrm{l}^{-1}, 0.09-0.91 \mathrm{mg} \mathrm{l}^{-1}$, and $0.01-0.83 \mathrm{mg} \mathrm{l}^{-1}$ respectively. $\mathrm{pH}$ was affected by water temperature. Variations in the $\mathrm{pH}$ values were due to changes in the values of free carbon dioxide, carbonate and bicarbonate in water (APHA, 2005). Dissolved oxygen was depended on water temperature (Boyd and Tucker, 1998; Manon and Hossain, 2011). High water temperature decreased dissolved oxygen and vice-versa (Lawson, 2011, Kataria et al., 1996). According to Huet (1975) suitable dissolved oxygen for rainbow trout culture in raceways was above $8 \mathrm{mg}^{-1}$. High free carbon dioxide decreased dissolve oxygen and vice-versa (Lawson, 2011). It showed seasonal changes being high in summer and monsoon and low during autumn and winter (Boyd and Tucker, 1998). Total alkalinity and total hardness was maximum during winter and minimum during summer that ranged $17-97 \mathrm{mg}^{-1}$ and 11-90 $\mathrm{mg}^{-1}$ respectively.

$\mathrm{NH}_{4}$ varied due to water temperature, dissolved oxygen and $\mathrm{pH}$ (Mannon and Hossain, 2011) and ranged from 0.25$0.35 \mathrm{mg} \mathrm{l}^{-1}$ (Chakraborty, 1998) and 0.008$0.028 \mathrm{mg} \mathrm{1}^{-1}$ (Acherjee and Barat, 2011). $\mathrm{NO}_{3}$ over $5 \mathrm{mg} \mathrm{l}^{-1}$ indicated pollution and became toxic at $30 \mathrm{mg}^{-1}$ (Lawson, 2011). Suitable range of $\mathrm{PO}_{4}$ was $0.200-0.308 \mathrm{mg}^{-}$ 1 (Kalwale and Savale, 2012), however, more than $0.7 \mathrm{mg} \mathrm{l}^{-1} \mathrm{PO}_{4}$ was harmful to fish (Boaventura et al., 1997).

Range of relative humidity (62.4-88.7) and rainfall $(0.0-503.4 \mathrm{~mm})$ was maximum in monsoon and minimum in winter season. Correlation coefficient (r) analyses of all the parameters are computed in Table 2 . Parameters of the first year were slightly higher than second due to fluctuation in temperature, velocity and discharge, relative humidity, and rainfall influenced by climatic factors, geography, seasons, and environment of the origin and occurrence of the water resource, thus affecting rest of the parameters. Water velocity and water discharge were maintained as per requirement of the culture.

\section{Acknowledgements}

Authors are thankful to Mr. Gyanendra Bahadur Karki, Chief, and Mr. Ram Hulas Jha, Laboratory Officer, KUKL Water Laboratory, Kathmandu, Nepal for providing necessary laboratory facilities for the present study. They are also thankful to Mr. Nand Kishor Roy, Senior Technical Officer, GoN, FRD, Godawari, Lalitpur, Kathmandu, Nepal for his cooperation and help during the study.

\section{References}

Acherjee, M.L. and S. Barat 2011. Spatio-temporal dynamics of physico-chemical factors of river Relli in Darjeeling Himalaya, West Bengal, India NBU J. Ani. Sci. 5: 24-33.

Adoni, A.D., G. Joshi, K. Ghosh, S.K. Chourasia, A.K. Vaishya and H.G. Verma 1985. Workbook on Limnology. Pratibha Publication, C-10, Garunagar, Sagar (MP).

American Public Health Association (APHA) 2005. Standard Methods for the Examination of Water and Waste Water $\left(21^{\mathrm{st}} \mathrm{Ed}\right.$.). USA.

Arain, M.B., T.G. Kazi, M.K. Jamali, H.I. Afridi, J.A. Baig, N. Jalbani and A.Q. Shah 2009. Pak. J. Anal. Environ. Chem. 9(2): 101.

Bardach, J.H., W.O. Ryther and W.O. McLarney 1972. Commercial culture of freshwater salmonids, genera Salmo, Thymallus and Hucho. 
Rakesh Prasad Bhagat and Sudip Barat / Our Nature (2015), 13(1): 50-57.

In The farming and husbandry of freshwater and marine organisms. John Willey and Sons. pp 397449.

Bartoli, M., D. Nizzali, D. Longhi, A. Laini and P. Viaroli 2007. Impact of a trout farm on the water quality of an Apennine Creek for daily budgets of nutrients. In Departmento de Scienze Ambien tali. Universitadegli di parma, Italy. pp 43-100. http://dx.doi.org/10.1080/02757540601084003

Boaventura, R., A.M. Pedro, J. Coimba and E. Lencastre 1997. Trout farm effluents: characterization and impact on the receiving streams. Environ. Poll. 95(3): 379-387. http://dx.doi.org/10.1016/S0269-7491(96)00117-0

Boyd, C.E. and C.S. Tucker 1998. Pond Aquaculture and Water Quality Management. Kulwer Academic Publishers, Boston.700p.

Chakraborty, D. 1998. Limnological studies on Lake Sinchel, a mountain lake in Darjeeling. Environment and Ecology 16: 31-35.

Huet, M. 1975. Breeding and cultivation of salmonids, a fish culture in cold water. In Textbook of Fish Culture, Breeding and Cultivation of Fish. Fishing News Books Ltd, England. pp 59-112.

Jacobsen, D. 2008. Tropical High-Altitudes. In Tropical Streams Ecology (Ed. D. Dudgeon). Academic Press, Elsevier, UK. pp 313-317. http://dx.doi.org/10.1016/B978-012088449$0.50010-8$

Kalwale, A.M. and P.A. Savale 2012. Determination of physico-chemical parameters in Deoli Bhorus
Dam water. Advances in Applied Science Research 3(1): 273-279.

Kataria, H.C., H.A. Quereshi, S.A. Iqbal and A.K. Shandilya 1996. Assessment of water quality of Kolar reservoir in Bhopal (MP). Pollution research 15(2): 191-193.

Lawson, E.O. 2011. Physico-chemical parameters and heavy metal contents of water from the mangrove swamps of Lagos lagoon, Lagos, Nigeria.

Advances in Biological Research 5(1): 8-21.

Manon, M.R. and M.D. Hossain 2011. Ecology of

Cyprinus carpio var. specularis (Physicochemical conditions of the habitat). J. Sci. Foundation 9(1-2): 133-139.

McGregor, G.R. and S. Nieuwolt 1998. Tropical climatology: an introduction to the climates of the low latitudes. Wiley Chichester, UK.

Pradhan, N., R.M. Mulmi and R.P. Dhakal $2007 / 2008$. Water quality parameters for Trout (Oncorhynchus mykiss) farming in hills of Nepal In Proceedings of the First National Workshop on Rainbow Trout Farming Scaling-up Strategies in Nepal.18-19 January, Kathmandu, Nepal. pp 1246.

Svobodova, Z., R. Lloyd, J. Machova and B. Vykusova 1993. Water quality and fish health. Technical Paper No. 54, EIFAC, FAO, Rome. 59 p.

Wetzel, G.W. 2001. Limnology: Lake and river ecosystems. New York Academic Press.

Yamazaki, T. 1991. Culture of foreign origin fishes. Farming Japan (25 ${ }^{\text {th }}$ Anniversary) 25(1): 41-46. 